\title{
Spatiotemporal transcriptomics reveals pathogenesis of viral myocarditis
}

Authors: Madhav Mantri ${ }^{1}$, Meleana M. Hinchman ${ }^{2}$, David W. McKellar ${ }^{1}$, Michael F. Z. Wang ${ }^{1}$, Shaun T. Cross $^{1,2,3}$, John S. L. Parker ${ }^{2,3^{*}}$, Iwijn De Vlaminck ${ }^{1,3^{*}}$

Affiliations: ${ }^{1}$ Nancy E. and Peter C. Meinig School of Biomedical Engineering, Cornell University, Ithaca, New York, ${ }^{2}$ Baker Institute for Animal Health, College of Veterinary Medicine, Cornell University, Ithaca, New York, ${ }^{3}$ Cornell Institute for Host-Microbe Interactions and Disease, Cornell University, Ithaca, New York.

*To whom correspondence should be addressed: id93@cornell.edu and jsp7@cornell.edu

\section{ABSTRACT}

11 A significant fraction of sudden death in children and young adults is due to viral myocarditis, an inflammatory disease of the heart. Here we used integrated single-cell and spatial transcriptomics to study the temporal, spatial, and cellular heterogeneity of host-virus interactions associated with reovirus-induced myocarditis in neonatal murine hearts. We further assayed the intestine, the primary site of reovirus infection to establish the cellular targets of the virus and the chronology of molecular events that ultimately lead to myocarditis. Our data give insight into the cell-type specificity of innate immune responses, and into the transcriptional states of inflamed cardiac cells that recruit circulating immune cells, including cytotoxic T cells which induce pyroptosis. Analyses of spatially restricted gene expression in myocarditic regions and the border zone identified immune-mediated cell-type specific injury and stress responses. Overall, we observe a complex network of cellular phenotypes and cellcell interactions associated with viral myocarditis. 
24

Viral infection is the most common cause of myocarditis ${ }^{1,2}$. The resulting inflammatory cardiomyopathy can lead to arrhythmias, dilated cardiomyopathy, and death ${ }^{1,3,4}$. In humans, viral myocarditis is challenging to study because of the low sensitivity of available diagnostic testing, the acute onset of the disease, the focal nature of the disease, and the extreme heterogeneity of immune-virus interactions in complex cardiac tissues ${ }^{4-6}$. In mice, mammalian orthoreovirus offers a flexible model system ${ }^{7}$. After oral inoculation, the Type 1 Lang (T1L) reovirus strain initially infects the gastrointestinal tract. Within days the infection then spreads to secondary sites in the body, including the heart, leading to myocarditis in up to $50 \%$ of infections ${ }^{7-9}$. Yet, even in this mouse model, the molecular pathogenesis of viral myocarditis is difficult to study because of the complex network of cardiac and immune cell types involved and the cellular, spatial, and temporal heterogeneity of the disease ${ }^{2,10}$. Consequently, neither the cell types that are responsible for the innate immune response, nor the cell types that are infected in vivo have been identified. Similarly, the responses of infected and uninfected bystander cells within the heart have not been characterized. In addition, the protective versus damaging effects of adaptive immune responses have not been quantified. Experiments in mice with severe combined immunodeficiency (SCID) indicate that adaptive immune responses are not required for myocardial injury and heart failure ${ }^{7,11}$, but these observations do not exclude the possibility that immune-cell-mediated injury is important in immunocompetent mice. Unbiased characterization of all cellular phenotypes as a function of time and location within infected cardiac tissues is needed to address these knowledge gaps.

Here we used integrated single-cell and spatially resolved RNA-sequencing (RNA-seq) to study the cellular and spatial heterogeneity of myocarditic processes in the hearts of reovirusinfected neonatal mice at multiple time points after infection. We also applied these technologies to study the innate response to reovirus infection in the intestine. In addition, we performed time-series single-cell RNA-seq (scRNA-seq) of cardiac tissues of mice infected with a reovirus point mutant that does not cause myocarditis. To establish viral tropism, we implemented molecular enrichment of non-polyadenylated viral transcripts that were otherwise poorly represented in the transcriptomes. Our measurements give insight into the cell-type specificity of innate immune responses, into the tropism of the virus in the intestine and the heart, and into the transcriptional states of cell types involved in the production of inflammatory cytokines and the recruitment of circulating immune cells. Analyses of spatially restricted gene expression in myocarditic regions and the border zone around those regions identified injury and stress responses in different cell types, including cardiomyocytes. Overall, our data identify spatially restricted cellular interactions and cell-type specific host responses during reovirus-induced myocarditis. 
RESULTS

Single-cell and spatial transcriptomics of reovirus T1L-infected neonatal mice hearts

To elucidate the pathogenesis of reovirus-induced myocarditis, we analyzed heart tissues collected from neonatal mice infected orally with either the T1L strain of reovirus or a mock control (Methods, Fig. 1A). We generated scRNA-seq data for 31,684 cells from infected hearts and mock controls at 4, 7, and 10 days post-infection (dpi), and 8,243 spatial transcriptomes for four tissue sections from infected hearts and mock controls at 4 and $7 \mathrm{dpi}$ from the same litter (10x Chromium and 10x Visium, Methods, Supp Fig. 1A-1B and Fig. 1B1C). The single-cell transcriptomes represented 18 distinct cell types, including cardiomyocytes, endocardial cells, cardiac fibroblasts, endothelial cells, mural cells, macrophages, neutrophils, NK cells, dendritic cells, T cells, and B cells (Methods, Fig 1B, Supp Fig 1C-E). Clustering of the spatial transcriptomic data revealed distinct transcriptional programs for myocarditic regions and the border zone surrounding the myocarditic regions in the 7 dpi reovirus-infected heart that corresponded to areas of tissue damage identified by H\&E staining (Fig. 1C). The combination of scRNA-seq and spatial transcriptomics allowed us to resolve and visualize cell types and gene expression in a spatial context (Methods, Supp Fig 1F). Because the virus first infects the gastrointestinal tract before it spreads to other body sites including the heart, we also performed scRNA-seq and spatial transcriptomics on ileum. We obtained 7,695 single-cell transcriptomes and 8,027 spatial spot transcriptomes for ileum from mock and infected samples at 1 and 4 dpi (Fig. 1D, Supp Fig. 2A-C).

To faithfully identify reovirus transcripts in the ileum and heart, which are not polyadenylated, we performed hybridization-based enrichment of viral fragments captured in the scRNA-seq libraries (Methods). In the ileum, we captured a total of 13,100 unique viral transcripts, with viral load decreasing from $1 \mathrm{dpi}$ to $4 \mathrm{dpi}$ (Supp Fig. 2D-2E). At $1 \mathrm{dpi}$, entero-endocrine cells had the highest fraction of infected cells followed by enterocytes and goblet cells, all of which are present in the gut epithelium. Lymphatic endothelial cells were infected at $4 \mathrm{dpi}$, suggesting that the virus reaches the bloodstream via lymphatic drainage to allow transmission of the virus to secondary sites in the body, including the heart, as shown before ${ }^{12}$ (Supp Fig. 2E). We captured 2,762 unique viral transcripts from 392 cells in the T1L-infected hearts. The viral load first increased from $4 \mathrm{dpi}$ to $7 \mathrm{dpi}$ and then decreased from $7 \mathrm{dpi}$ to $10 \mathrm{dpi}$, consistent with viral titer assays performed on whole hearts ${ }^{9,13}$ (Fig. 1E, Supp Fig. 2F-2G). Endocardial and endothelial cells were the most frequently infected cell types at $4 \mathrm{dpi}$, suggesting that endocardial cells lining the ventricular lumen and endothelial cells lining the cardiac vasculature are among the first cells to be infected in the heart (Fig. 1E). We detected viral transcripts in neutrophils, dendritic cells, and T cells in the 7 dpi heart (Fig. 1E). This observation suggests that antigen-presenting cells and immune cells may contribute to the spread of infection to other organs in the body. The role of infected dendritic cells in bringing more reovirus to the cardiac tissue during systemic infection has been discussed previously ${ }^{8}$. 


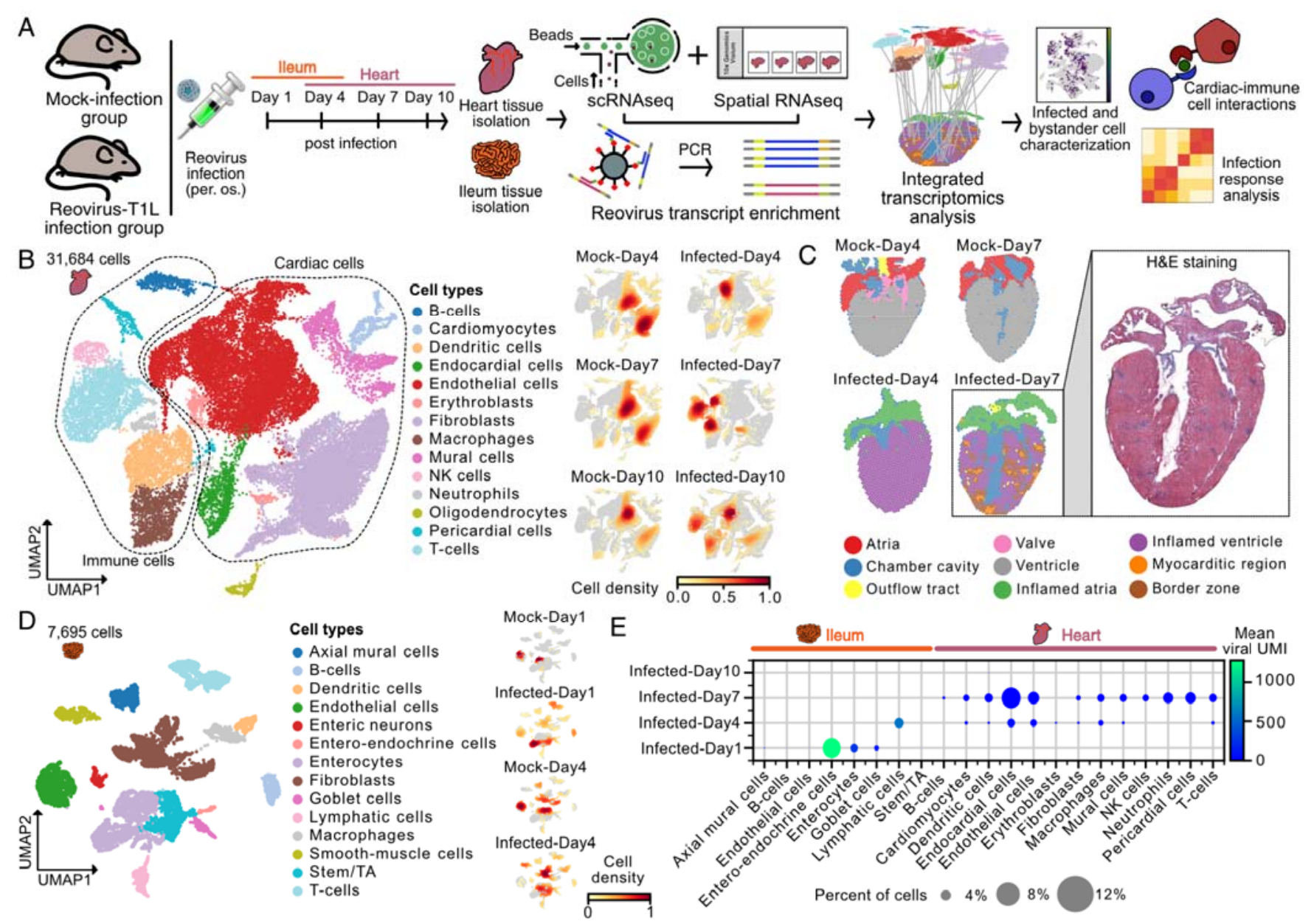

Figure 1: Single-cell and spatial transcriptomics of cardiac and ileum tissue of reovirus-infected neonatal mice. A) Experiment and analysis workflow. B) UMAP plot of 31,684 single-cell transcriptomes from mockinfected and reovirus-infected hearts at 4,7 , and $10 \mathrm{dpi}$, clustered by gene expression and colored by cell type (left). UMAP plots showing the gaussian kernel density of cells across samples for the heart scRNA-seq data (right). C) 8,243 spatial transcriptomes of cardiac tissue sections from mock-infected and reovirus-infected mice at 4 and 7 dpi. Haematoxylin and Eosin (H\&E) stained image of reovirus-infected myocarditic tissue section used for spatial transcriptomics at 7 dpi (in box). D) UMAP plot of 7,695 single-cell transcriptomes from mock-infected and reovirus-infected ileum at day 1 and $4 \mathrm{dpi}$, clustered and colored by cell type (left). UMAP plots showing the gaussian kernel density of cells across samples for the ileum scRNA-seq data (right). E) Dot plot showing the percent of cells with non-zero viral transcripts and the mean viral transcript counts (UMIs) in ileal and cardiac cell types.

\section{Endothelial cells are primed with basal interferon response and play an important role in initiating host innate immune response}

To detect early transcriptional differences in the cardiac tissue after infection, we performed Differential Gene Expression Analysis (DGEA, mock vs infected hearts at 4 dpi, Methods). This analysis revealed a significant upregulation of 226 genes in the infected heart (two-sided Wilcoxon test, log fold-change $>1.0$ and $p$-value $<0.01$ ), including genes related to the interferon- $\beta$ pathway, interferon signaling, and innate immune responses (Supp Fig 3A-3B, Fig. 2A). 
120 To quantify and compare the overall magnitude of early infection responses across different 121 cell types, we computed a gene module score (infection response score, IR, module of 226 122 genes selected above). Comparison of the IR of different cell types in absence of infection 123 revealed a small, but higher IR in endothelial cells as compared to other cardiac cell types 124 (Fig. 2B). In response to infection, an increase in IR was observed for all cardiac cell types, 125 but the greatest increase in IR was observed for endothelial cells (Fig. 2B). These data 126 suggest that endothelial cells lining the cardiac vasculature are important initiators of the host 127 defense to viral infection. Comparison of IR scores using the spatial transcriptomic data shows 128 increased IR scores in the infected hearts at 4 and 7 dpi with the highest scores found in 129 myocarditic regions (Fig. 2C). Given our observation that endothelial cells within the heart had 130 the highest IR score in absence of infection, we asked if this observation was unique to heart 131 tissue or was a more general phenomenon. To this end, we used the Tabula Muris scRNA-seq 132 mouse atlas ${ }^{14}$ and estimated the IR of $\sim 16,000$ cells of five major cell types (epithelial cells, 133 fibroblasts, endothelial cells, smooth muscle cells, and mesenchymal cells) across 10 different 134 organs and tissues. This analysis revealed that endothelial cells consistently had the highest 135 IR score across all tissues in mice (Fig. 2D). These results indicate that endothelial cells lining 136 the vasculature have a higher basal expression of innate response genes within most tissues, which may prime these cells to respond to viral dissemination within the blood and lymphatics.

138

To investigate the cell-type-specific IR in the ileum, the primary site of reovirus infection, we performed DGEA on reovirus-infected at 1 dpi compared to mock-infected ileal cells and found a significant upregulation of 438 genes (two-sided Wilcoxon test, log fold-change $>1.0$ and $p$ value $<0.01$ ), related to the interferon-beta pathway, interferon signaling, and innate immune responses (Supp Fig. 3C-3D). We computed an IR score using this module of 438 genes and observed higher basal IR scores in enterocytes and entero-endocrine cells as compared to other ileal cell types (Fig. 2E). Enterocytes further showed the highest increase in IR score after infection, followed by entero-endocrine, endothelial, and lymphatic cells (Fig. 2E). Comparison of IR scores for spatial transcriptomic data further supported our analysis of the scRNA-seq data, showing increased IR scores in the infected ileum at 1 and 4 dpi with the highest scores evident within intestinal mucosa and villi (Fig. 2F). The intestinal epithelial cells must tolerate commensal microorganisms present in the lumen of the gut and yet still be responsive to invasive pathogens. Our data suggest that to achieve this, enterocytes and entero-endocrine cells in the gut epithelium are primed with a basal interferon response and play an important part in mounting innate immune responses in the early stages of viral infection. 
A

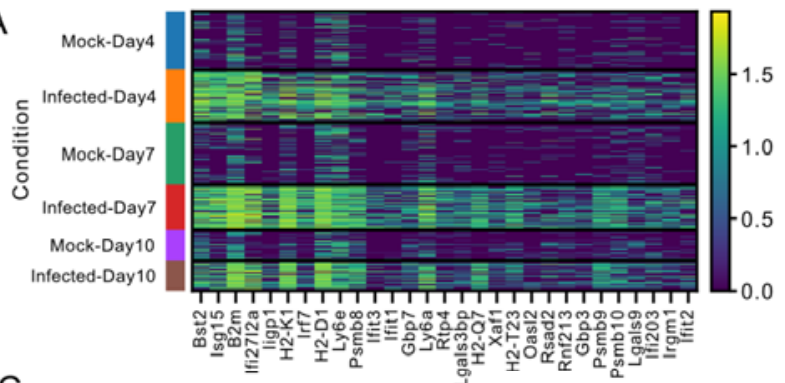

C Mock-Day4 Mock-Day7

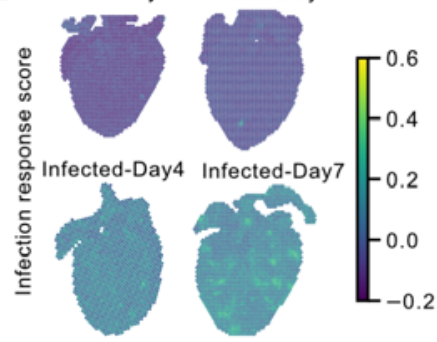

E

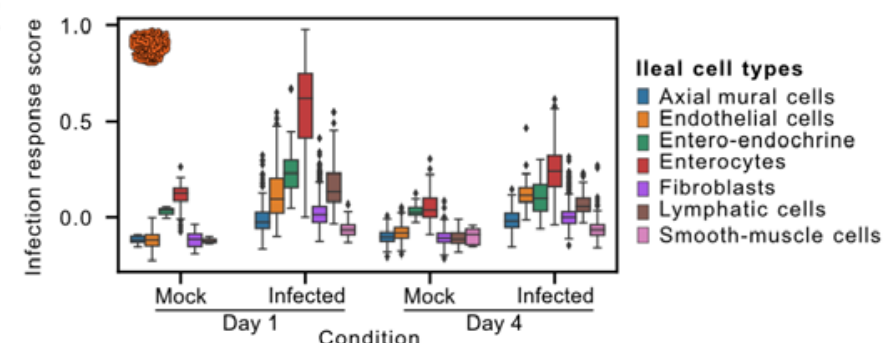

Condition

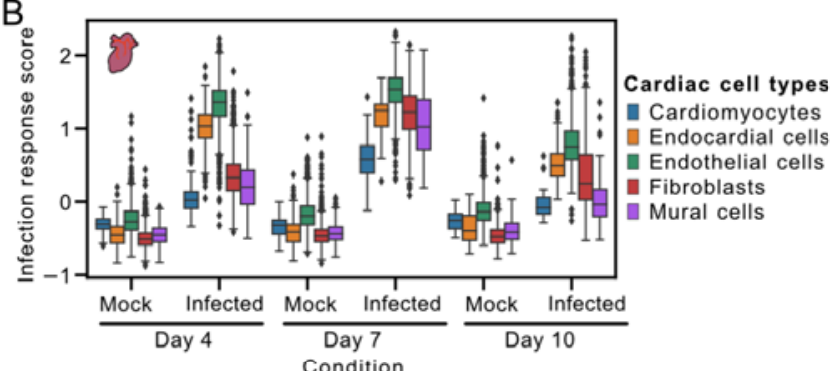

D

Condition

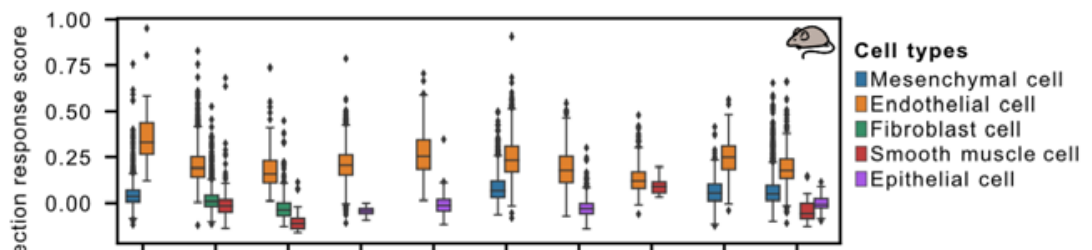

Figure 2: Endothelial cells have the highest basal interferon response and the highest increase in innate response upon reovirus infection $A$ ) Heatmap showing the expression of the 25 most upregulated genes in the reovirus-infected heart as compared to mock at $4 \mathrm{dpi}$. B) Infection response score for cardiac cell types in scRNAseq data across mock-infected and reovirus-infected hearts at three distinct stages. The infection response score represents the gene module score for a panel of 226 genes that are significantly upregulated (two-sided Wilcoxon test, log fold-change $>1.0$ and $p$-value $<0.01$ ) in the reovirus-infected heart as compared to the mock-infected heart at 4 dpi. C) Infection response score (defined above) across spots in spatial transcriptomics data. D) Infection response score calculated for five common cell types across 13 tissues from the tabula-muris mouse atlas data. E) Infection response score for ileal cell types in scRNA-seq data across mock-infected and reovirusinfected ileum at two distinct stages. The infection response score represents the gene module score for a panel of 438 genes significantly upregulated (two-sided Wilcoxon test, log fold-change $>1.0$ and $p$-value $<0.01$ ) in the reovirus-infected ileum at $1 \mathrm{dpi}$ as compared to the mock-infected ileum. F) Infection response score for spatial transcriptomics data from mock-infected and reovirus-infected ileum at two distinct stages.

\section{Cytotoxic T cells recruited by inflamed endothelial cells induce pyroptosis}

To explore the heterogeneity of endothelial cell phenotypes in more detail, we reclustered all 9,786 cardiac endothelial cells in the scRNA-seq data. We observed four distinct phenotypes: i) uninflamed venous endothelial cells expressing Nr2f2 and Aplnr mainly derived from the mock controls, ii) arterial endothelial cells expressing Gja4, Gja5, and Cxc/12 derived from both mock and infected cardiac hearts, iii) inflamed endothelial cells derived from infected hearts at 4 and $10 \mathrm{dpi}$, and iv) inflamed endothelial cells from the heart at $7 \mathrm{dpi}$, with both inflamed endothelial cell clusters expressing Isg15, ligp1, and Ly6a (Fig. 3A-B). DGEA across endothelial subclusters revealed that the inflamed $7 \mathrm{dpi}$ endothelial cells overexpressed chemokines $\mathrm{Cxc} / 9$ and $\mathrm{Cxc} / 10$, which are generally involved in immunoregulatory and 
178 inflammatory processes, but more specifically in the recruitment of T cells (Fig. 3B-3C, Supp 179 Fig. 3E). In line with this observation, T cells in the 7 dpi hearts expressed the $C x c r 3$ receptor 180 (see below). The Cxc/9-high inflamed endothelial cells furthermore expressed high levels of 181 cell adhesion marker genes Vcam1 and Icam1, which help immune cells in the blood to attach to endothelial cells ${ }^{15}$ (Fig. 3C, Supp Fig. 3E, 3I). The endothelial cells also overexpressed MHC class 1 (H2-D1 and H2-K1) and MHC class 2 (Cd74) molecules, suggesting their involvement in antigen presentation to adaptive immune cells (Fig. 3B-C, Supp Fig. 3E). Endothelial cells have been shown to be involved in antigen presentation and shaping the cellular immune response in infectious myocarditis ${ }^{15}$. Gene ontology (GO) term enrichment analysis identified pathways further supporting the $C x c / 9$-high endothelial cells' involvement in leukocyte cell-cell adhesion, $T$ cell activation, regulation of interleukin-8 production, and response to cytokines, interferon-gamma, interleukin-1, and tumor necrosis factors (Supp Fig. $3 F)$.

The observation that endothelial cells are involved in the recruitment of $\mathrm{T}$ cells prompted us to explore the heterogeneity of $T$ cells in the infected hearts in more detail. To this end, we reclustered 2,205 $\mathrm{T}$ cell single-cell transcriptomes, leading to four subclusters representing three $T$ cell subtypes, i) $C d 8+$ cytotoxic $T$ cells, ii) $C d 4+$ helper $T$ cells, and iii) naive $T$ cells (Fig 3E-F). Both the cytotoxic and helper $\mathrm{T}$ cells identified within infected hearts expressed Cxcr3 receptor, interferon-gamma (Ifng), and the chemokines Cc/3, Ccl4, Ccl5, S100A4, and S100A6, suggesting their involvement in neutrophil recruitment and activation (Fig. 3G, Supp Fig. 3G). The $C x c r 3$ receptor binds selectively to the chemokines $C x c / 9$ and $C x c / 10$, promoting chemotaxis (Fig. 3G). Cytotoxic $T$ cells represented the majority of infiltrating $T$ cells and expressed Prf1, Gzma, Gzmb, and Gzmk, coding for lytic molecules associated with the granzyme-dependent exocytosis pathway ${ }^{16}$ (Fig. 3F-G, Supp Fig. 3G, 3K). These cells also expressed tumor necrosis factor superfamily genes Fas/ and Tradd, which are involved in the Fas-induced cell death pathway. Fas/ binds to Fas on the surface of target cells and mediates programmed cell death signaling and NF-KB activation (Fig. 3G). The Fasl-Fas apoptosis pathway is important in regulating $T$ cells, in promoting tolerance to self-antigens, and is a mechanism by which cytotoxic T cells kill target cells ${ }^{16}$. GO term enrichment analysis identified pathways involved in neutrophil activation and degranulation, processing and presentation of exogenous peptide antigen, interleukin-1-mediated signaling pathway, tumor necrosis factormediated signaling, NIK/NF-kappaB signaling, cellular response to lectin, and apoptotic processes (Supp Fig. 3H).

The downstream gene markers for cell death-associated pathways Pycard, Acer2, Zbp1, and Caspases Casp1, Casp4, and Casp12 were enriched in the Cxcl9-high endothelial cells. This raises the possibility that cytotoxic lymphocytes are responsible for inflamed endothelial cell death (Fig. 3B-3C, Supp Fig. 3I). GO term enrichment of endothelial cells confirmed an upregulation of cell death pathways including activation of cysteine-type endopeptidase activity involved in the apoptotic process, positive regulation of extrinsic apoptotic signaling pathway, and pyroptosis pathway (Supp Fig. 3F). We assessed the spatial transcriptomic data to 
218 validate direct interactions between Cxc/9-high inflamed endothelial cells and T cells and found 219 that they were indeed spatially co-localized in the myocarditic regions and the border zone 220 (Supp Fig. 1F). We calculated gene module scores for genes associated with ontology terms 221 enriched in Cxcl9-high endothelial and cytotoxic $\mathrm{T}$ cells for spatial transcriptomics data and 222 found these pathways to be enriched in the myocarditic regions (Fig. 3D, 3H, and Supp Fig. 223 3I-3L). Collectively, these results suggest that endothelial cells lining the cardiac vasculature 224 act as a blood-heart barrier and play an important role in the recruitment and activation of the 225 host adaptive immune system. These cells may be the target of both direct viral damage and 226 immune-mediated damage during reovirus-induced myocarditis. Damage to the 227 microvasculature within the heart may then cause loss of blood supply and be a factor in the 228 subsequent death of cardiomyocytes independent of direct viral replication.
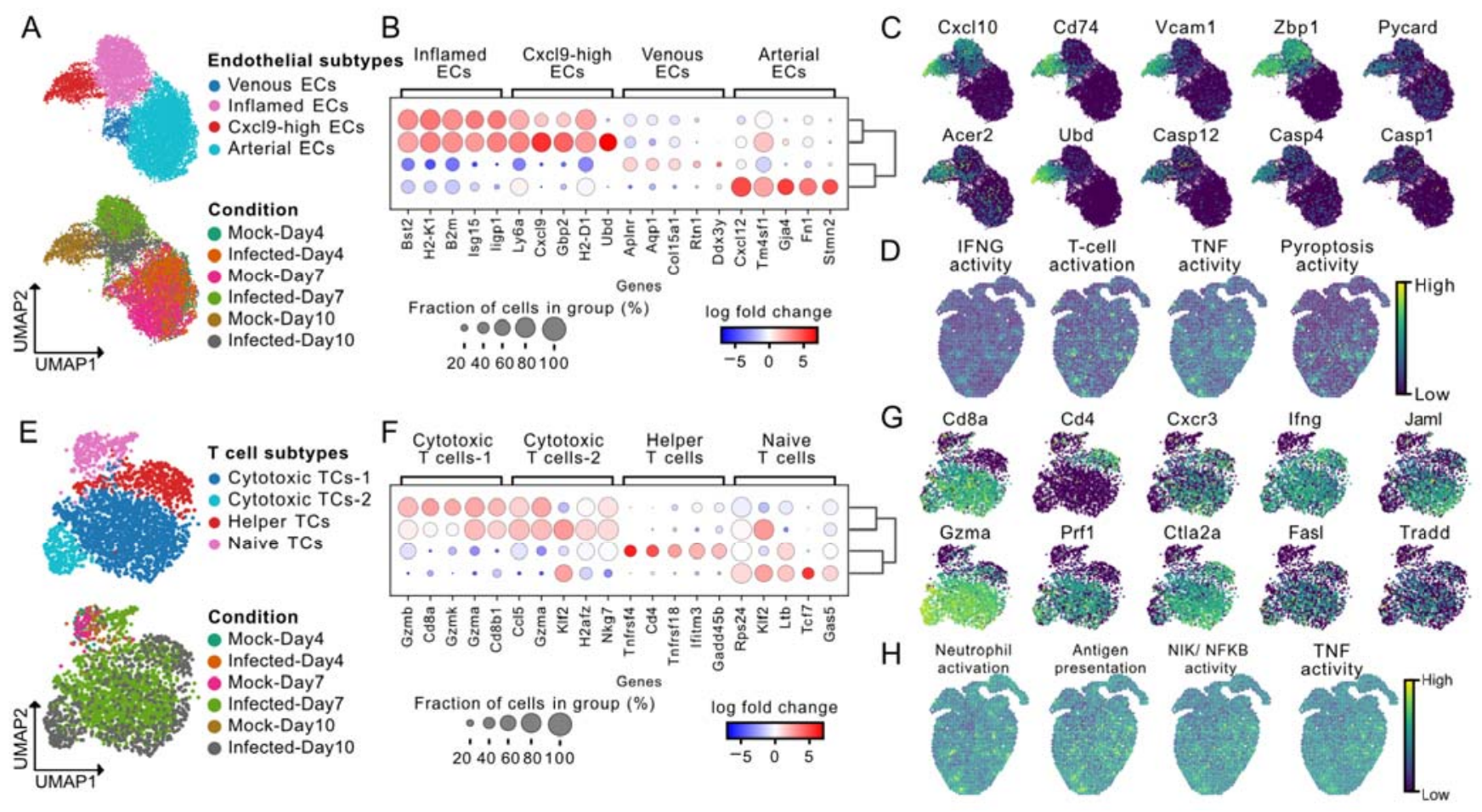

Figure 3 Cytotoxic T cells recruited by inflamed endothelial cells induce pyroptosis in myocarditic tissue A) UMAP plot of 9,786 single-cell endothelial cell transcriptomes from mock-infected and reovirus-infected hearts at 4, 7, and 10 dpi colored by endothelial cell (EC) subtype clusters (phenotypes) (top) and condition (bottom). B) Heatmap showing top-five differentially expressed genes (two-sided Wilcoxon test, log fold-change $>1.0$ and $p$ value $<0.01$ ) for endothelial cell subtypes. C) UMAP plot showing the expression of genes upregulated in CxCl9high endothelial cells. D) Spatial transcriptomic maps of cardiac tissue from reovirus infected hearts at $7 \mathrm{dpi}$ showing gene module scores calculated for four GO terms enriched in Cxcl9-high endothelial cells. E) UMAP plot of 2,205 single-cell T cell (TC) transcriptomes from mock-infected and reovirus-infected hearts at 4, 7, and $10 \mathrm{dpi}$ colored by $\mathrm{T}$ cell subtype clusters (top) and condition (bottom). F) Heatmap showing top-five differentially expressed genes (two-sided Wilcoxon test, log fold-change $>1.0$ and $p$-value $<0.01$ ) for T cell subtypes. G) UMAP plot showing the expression of genes upregulated in cytotoxic $T$ cells from myocarditic heart at $7 \mathrm{dpi}$. H) Spatial transcriptomics maps of cardiac tissue from reovirus infected hearts at $7 \mathrm{dpi}$ showing gene module scores calculated for four GO terms enriched in cytotoxic T cells. 


\section{Spatially restricted gene expression in myocarditic tissue}

245

246

247

248

249

250

251

252

253

254

255

256

257

258

259

260

261

262

263

264

265

266

267

268

269

270

271

272

273

274

275

276

277

278

279

280

281

282

The spatially restricted nature of myocarditis motivated us to explore the spatial heterogeneity of gene expression in reovirus-infected hearts. Our initial clustering of the spatial transcriptomic data revealed distinct transcriptional programs for myocarditic regions, the tissue bordering these myocarditic regions, and the rest of the ventricular tissue (Fig. 1C, 4A). Differential spatial gene expression analysis for these regions revealed upregulation in the myocarditic regions of cell-type markers for infiltrating immune cells, (Cd8a and Gzma for T cells, Nkg7 for NK cells, S100a8 for neutrophils), markers of inflammation (Cd52 and Lyc62, Supp Fig. 4A), and chemokines and cytokines ( $C \mathrm{cl} 5, \mathrm{Ccl} 2, \mathrm{Cxcl9}$, and $\mathrm{Cxc} / 10$ ). Analysis of the corresponding scRNA-seq data showed that $\mathrm{Ccl} 5$ is expressed by dendritic cells, $\mathrm{Ccl} 2$ by fibroblasts, and $\mathrm{Cxc} / 9$ and $\mathrm{Cxc} / 10$ by endothelial cells. The receptor for Ccl2, Ccr2, is expressed in macrophages, indicating that fibroblasts use the Ccl2-Ccr2 axis for macrophage recruitment during myocardial inflammation, as described recently ${ }^{17,18}$ (Supp Fig. 4A-B). Collectively these analyses show that chemokine-producing endothelial and cytokineproducing fibroblast cells recruit immune cells to the myocarditic tissue.

Closer inspection of the myocarditic regions and border zones showed an upregulation of additional genes of interest, including Timp1, AW112010, Clu, Ankrd1, Gm4841, and Ctss (Fig. 4B). Timp1 is mainly expressed by inflamed fibroblasts in the scRNA-seq data (Supp Fig. 4D). Timp1 is a natural inhibitor of the matrix metalloproteinases (MMPs), a group of peptidases involved in the degradation of the extracellular matrix. Upregulation of Timp1 in patients with deteriorating heart failure was reported previously ${ }^{19}$. AW112010 is expressed by inflamed endothelial cells and fibroblasts in the scRNA-seq data and was previously found to code for an interferon-induced small secreted protein with a crucial role in the innate immune response to infection and inflammation ${ }^{20}$. Clu is expressed in a subset of inflamed cells from all cardiac cell types in our data and was previously shown to be upregulated during severe myocarditis $^{21}$ (Supp Fig. 4D). Ctss, expressed mainly in monocytes, encodes a protease used for degradation of antigenic proteins to peptides for presentation on MHC class II molecules. Increased formation of immunoproteasomes in susceptible mice has been shown to affect the generation of antigenic peptides and subsequent $T$ cell activity in viral myocarditis ${ }^{22,23}$. GO term analysis of genes upregulated in the border zone revealed enrichment of terms related to the response to tumor necrosis factor, response to interleukin-1, and NIK/ NF-KB signaling (Supp Fig. 4E).

To further understand the effect of immune cell infiltration on the cell type composition around the myocarditic regions, we assessed cell type proportions as a function of distance from myocarditic regions in the tissue. We quantified the cell type proportions in myocarditic regions, the border zones, and the rest of the ventricular tissue, and found that the fraction of Cxc/9-high endothelial cells, Ccl2+ fibroblasts, T cells, dendritic cells, and NK cells was increased in the myocarditic regions, and the fraction of cardiomyocytes was reduced in myocarditic regions (Fig. 4C, Supp Fig. 1F). 
284 To understand the phenotype of $\mathrm{Ccl} 2+$ fibroblasts enriched in myocarditic region and border 285 zone, we reclustered 9,192 fibroblast cells from the scRNA-seq dataset and identified a distinct 286 cluster of inflamed Ccl2+ fibroblasts from the infected heart at 7 dpi (Supp. Fig. 4G-4H). The $287 \mathrm{Ccl} 2+$ fibroblast cells expressed high levels of $\mathrm{MHC}$ class 1 (H2-D1 and H2-K1), adhesion 288 marker genes Vcam1 and Icam1, and other genes like Serpina3g, C3, and Ms4a4d (Supp Fig. 289 4I). Moreover, these fibroblast cells also expressed Casp1 and Casp4, suggesting pyroptosis 290 activity in response to cytotoxic T cells (Supp Fig. 4I).

291 To investigate the effect of inflammation on cardiomyocytes in myocarditic hearts, we 292 reclustered 502 cardiomyocyte cells from the scRNA-seq dataset and identified three distinct 293 phenotypes: i) ventricular myocytes expressing Myl2, Myl3, and Mb derived from mock and 294 infected hearts at 4 and $10 \mathrm{dpi}$, ii) atrial myocytes expressing markers Myl4, Myl7, and Nppa 295 derived from mock and infected hearts at 4 and $10 \mathrm{dpi}$, and iii) inflamed myocytes from the 296 infected heart at 7 dpi expressing innate immunity genes Isg15, lgtp, and ligp1 (Fig. 4D-E). 297 Inflamed myocytes from the infected heart at $7 \mathrm{dpi}$ had a distinct phenotype as compared to 298 the myocytes from hearts at 4 and $10 \mathrm{dpi}$, which clustered with myocyte cells from mock299 infected hearts (Fig. 4E). To find transcriptional signatures for myocytes present in the border 300 zone, we selected genes that were both enriched in cardiomyocyte cells in the scRNA-seq 301 data and upregulated in the border zone. This analysis revealed that cardiomyocytes in the 302 border zone expressed Gm4841, Gm12185, Mt1, Mt2, Ankrd1, and Nppb (Fig. 4F, Supp Fig. 303 4F). Gm4841 and Gm12185 are interferon-inducible genes produced in response to interferongamma. Mt1 and Mt2 genes modulate inflammation and support remodeling in ischemic cardiomyopathy in mice ${ }^{24}$. Upregulation of Ankrd1, a myocyte survival factor, occurs during late-stage heart disease in patients with idiopathic dilated cardiomyopathy ${ }^{25}$. A recent study shows that cardiomyocytes expressing Ankrd1 are localized in the border zone on day 1 postmyocardial infarction ${ }^{26}$. Together, our analysis reveals that tissue injury is localized to myocarditic regions with remodeling programs being active in the border zone and demonstrates the importance of spatially resolved molecular measurements to study viral myocarditis. 

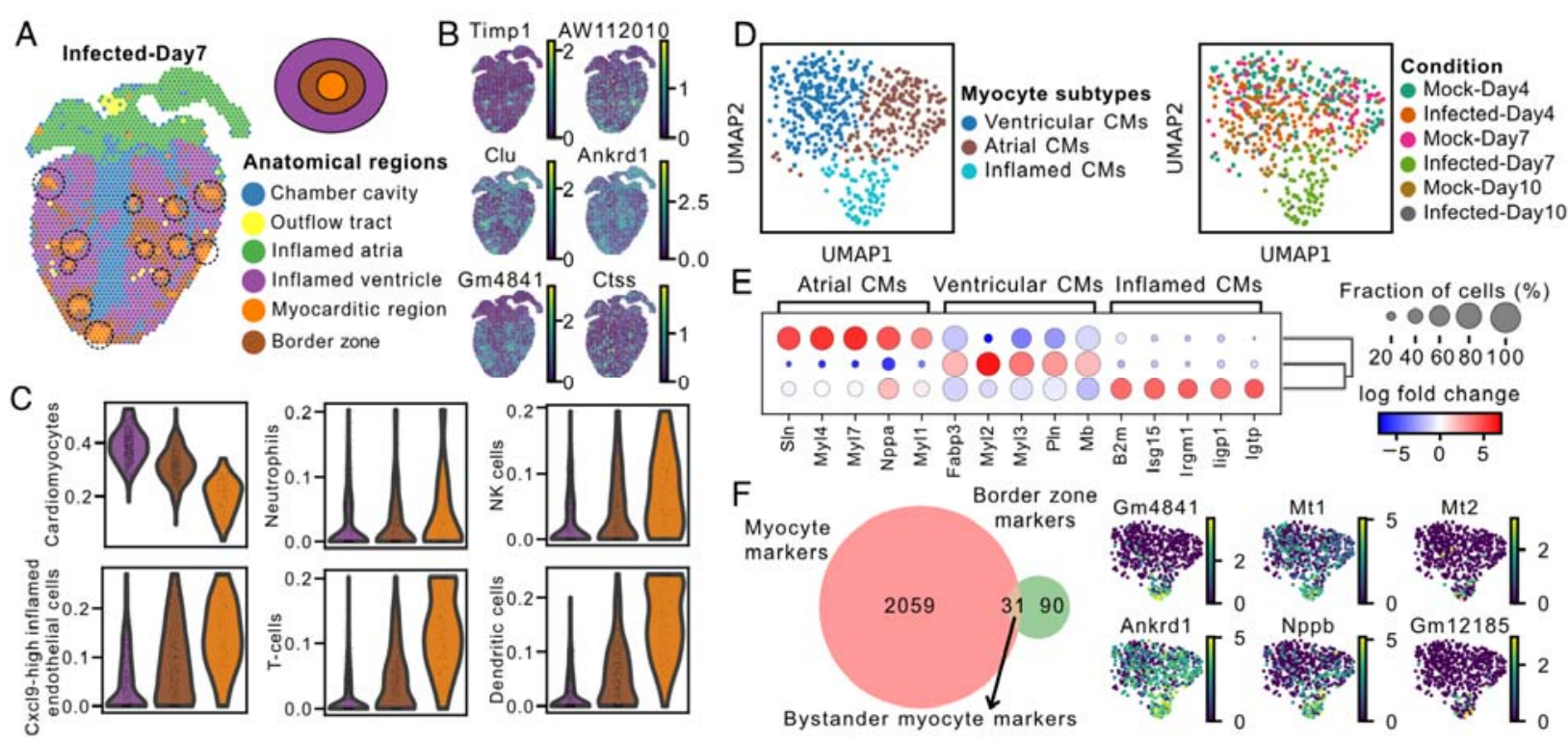

Fraction of cells $(\%)$

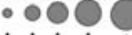

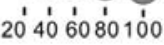
log fold change

$\begin{array}{lll}-5 & 0 & 5\end{array}$

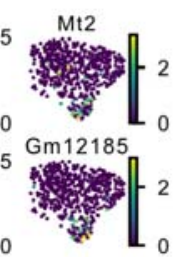

Figure 4 Myocarditic regions and the border zone have distinct transcriptomic profiles and cell type specific signatures. A) Spatial transcriptomics map of cardiac tissue section from reovirus-infected mice at $7 \mathrm{dpi}$ colored by spot clusters representing transcriptionally distinct tissue regions. B) Spatial transcriptomics maps of cardiac tissue sections from reovirus-infected mice at 7 dpi showing the expression of differentially expressed genes of interest in the myocarditic and the border zone. C) Changes in average predicted cell-type proportions across the infected ventricle, for cell types enriched in the myocarditic region and the border zone. D) UMAP plot of 502 single-cell cardiomyocyte cell transcriptomes from mock-infected and reovirus-infected hearts at 4, 7, and 10 dpi colored by myocyte cell subtype (phenotypes) (left) and condition (right). E) Heatmap showing top-five differentially expressed genes (two-sided Wilcoxon test, log fold-change $>1.0$ and $p$-value $<0.01$ ) for cardiomyocyte cell subtypes. F) Venn Diagram showing myocyte-specific genes upregulated in the border zone around the myocarditic regions (left). UMAP plot showing the expression of myocyte-specific genes which are upregulated in the border zone of myocarditic regions (right).

\section{Reduced adaptive immune cell infiltration associated with reovirus K287T mutant}

We recently reported a reovirus mutant T1L S4-K287T (K287T) which has a point mutation in the S4 gene encoding outer capsid protein sigma-3 ( $\sigma 3)$, a double-stranded (ds) RNA-binding multifunctional protein that promotes viral protein synthesis and facilitates viral entry and assembly ${ }^{9}$. K287T successfully infects the heart but produces lower viral titers relative to reovirus wild-type (WT) and does not cause myocarditis ${ }^{9}$. To validate our findings, we performed additional scRNA-seq for K287T infected hearts at 4, 7, and $10 \mathrm{dpi}$. We generated a total of 16,771 single-cell transcriptomes and integrated the data with the data from the wildtype (WT) virus. K287T-infected cells clustered with WT-infected cells for almost all cell types (Fig. 5A, Supp Fig. 4J). We performed viral transcript enrichment and compared the mean viral transcripts in WT- and mutant-infected cells. We found similar levels of viral transcripts for WT and K287T viruses at 4 dpi but a 60-fold lower viral load for K287T at $7 \mathrm{dpi}$, consistent with viral titer assays ${ }^{9}$ (Supp Fig. 4K-4L). We then compared early host responses among cardiac cell types due to K287T and WT infection. K287T induced a similar level of innate immune responses as WT reovirus with endothelial cells showing the highest increase in cardiac IR score (as defined before) at 4 dpi (Fig. 5B). We analyzed the cell type composition of Cxc/9high endothelial cells and immune cells between K287T- and WT-infected hearts and found 
that the 7 dpi K287T-infected heart at 7 dpi had significantly fewer $C x$ Cl9-high endothelial cells and immune cells infiltrating the heart compared to WT-infected heart. These differences are in line with the reduced levels of inflammation and tissue injury related to the K287T mutant (Fig. 5C). Our results show that cardiac endothelial cells mount a potent and robust innate immune response even when infected with the K287T mutant virus with reduced replication in most cell types. Clearance of the virus from the infected cells by $7 \mathrm{dpi}$ and a weaker adaptive host response then leads to the non-myocarditic phenotype of mutant K287T-infected hearts.

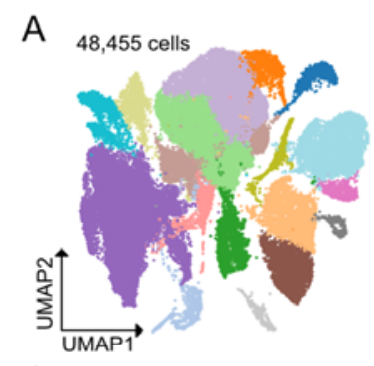

C

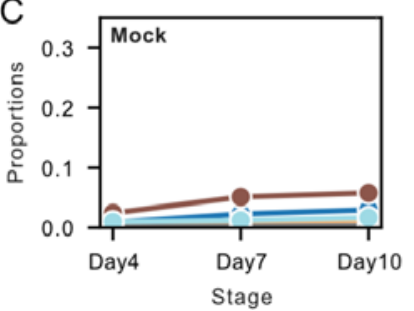

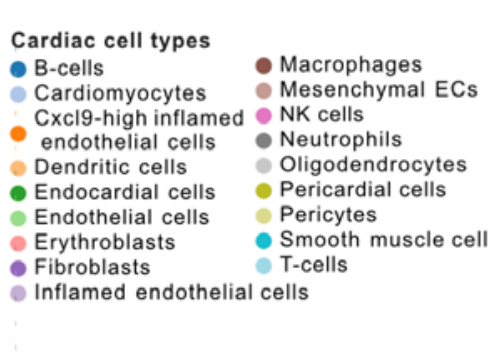

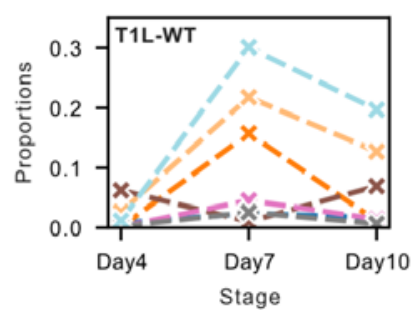

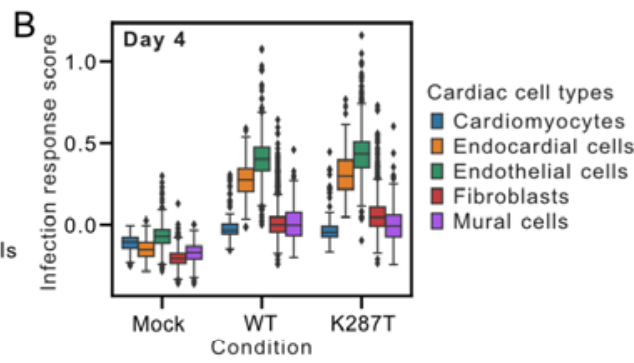

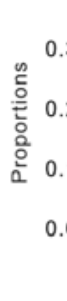

Cardiac cell types - B-cells Cxcl9-high inflamed endothelial cells - Dendritic cells - Macrophages - NK cells - T-cells

Figure 5 A robust innate immune response but reduced adaptive immune cell infiltration explains the nonmyocarditic phenotype on infection with reovirus K287T mutant. A) UMAP plot of 48,455 single-cell cell transcriptomes from mock-infected, reovirus-wildtype (WT) infected, and reovirus mutant (K287T) infected hearts at 4, 7, and 10 dpi colored by cell-type clusters. B) Infection response score for cardiac cell types in scRNA-seq data across mock-infected, reovirus-WT infected, and reovirus-K287T infected hearts on $4 \mathrm{dpi}$. The infection response score represents the gene module score for a panel of 226 genes that are significantly upregulated in the reovirus-WT infected sample as compared to the mock-infected sample at $4 \mathrm{dpi}$. C) Changes in cell-type proportions with time for cell types detected in the myocarditic regions. Panels show the changes in cell-type proportions across mock-infected, reovirus-WT infected, and reovirus-K287T infected cells.

\section{DISCUSSION}

Viral myocarditis has been recognized as a cause of heart failure for more than 50 years, but it is still a challenging disease to study, diagnose, and treat ${ }^{27}$. Here, we used integrated spatial and single-cell RNA-seq to dissect the temporal, spatial, and cellular heterogeneity of reovirusinduced acute myocarditis in a neonatal mouse model. We assayed ileum and heart tissues at multiple time points after infection. We investigated the cell types that are infected, and the cellular and spatial heterogeneity of innate and adaptive immune responses. We generated a total of thirteen scRNA-seq and eight spatial transcriptomics datasets, spanning two organs, four time points, and three infection conditions. Our data provide detailed insight into the chronology of molecular events that lead to reovirus-induced myocarditis. After oral inoculation, reovirus T1L infects entero-endocrine and enterocyte cells in the gut epithelium within $1 \mathrm{dpi}$. These cells mount a potent innate immune response to inhibit viral replication. The virus then infects the gut lymphatic cells within $4 \mathrm{dpi}$ and through lymphatic drainage, the 
372 virus is transmitted via the bloodstream to secondary sites in the body, including the heart. 373 Around $4 \mathrm{dpi}$, the virus infects the endothelial cells lining the cardiac vasculature. Endothelial cells mount a potent innate immune response in the heart. In symptomatic cases, inflamed endothelial cells secrete chemokines to recruit circulating immune cells, including cytotoxic $T$ cells. Tissue-infiltrating cytotoxic $T$ cells then induce pyroptosis in the myocarditic tissue. Overall, our experiments reveal a dynamic and spatially heterogeneous network of cellular phenotypes and cell-cell interactions associated with reovirus-induced myocarditis.

379 Integrated high-throughput scRNA-seq and spatial transcriptomics was recently used to study 380 heart development ${ }^{28,29}$ and heart disease ${ }^{26,30}$, but these methods have not been used to study viral myocarditis prior to our work. Bulk RNA-seq has been used previously to profile transcriptomic signatures of infection, inflammation, and tissue injury associated with viral myocarditis $9,31,32$. Yet, these ensemble-level approaches do not capture the cellular and spatial heterogeneity of host response to infection. scRNA-seq has recently been used to study Coxsackievirus B3 (CVB3)-induced myocarditis in a mouse model ${ }^{33}$. Lasrado et al. report inflammatory phenotypes of myeloid cells, the role of fibroblasts in remodeling and inflammation, and the role of cytotoxic T-cells in CVB3-induced myocarditis. However, the cardiac cell types that are targeted by the virus, the cell type heterogeneity in basal interferon response and innate immune response, and the spatial restriction of transcriptional programs were not explored in this study.

Previous studies have claimed that the direct cytopathic effect of viral replication on cardiac cells is the main cause of cardiac damage during reovirus-induced myocarditis ${ }^{7,34}$. Notably, Sherry et al. found that reovirus infection can induce myocarditis in immunodeficient mice lacking $B$ and/or $T$ cells, suggesting that reovirus-induced myocarditis does not strictly require adaptive immunity ${ }^{7,11}$. However, these previous experiments do not rule out the possibility that the host adaptive immune response can augment or delimit the nature and amount of host damage in immune-competent mouse, as is suggested by our work. Holm et al. and Stewart et al. have studied the protective role of innate immune responses in reovirus-induced myocarditis ${ }^{13,35}$. However, the temporal, spatial, and cell type heterogeneity of basal type-I IFN and innate immune responses to infection had not been characterized before our work. Miyamoto et al. and Stewart et al. compared basal levels of type-I IFN between cardiac myocytes and fibroblasts in vitro but these studies did not include all the cell types that make up complex cardiac tissues ${ }^{36,37}$.

Spatiotemporal characterization of viral myocarditis is crucial to understanding the viral and host factors that are important for disease pathology. This knowledge may ultimately lead to novel diagnostic approaches and better treatments. Several viruses that frequently infect humans can cause myocarditis, including Adenovirus, enteroviruses, Epstein-Barr virus, 409 implemented here can be used in future studies to investigate how the induction, 410 pathophysiology, and course of myocarditis induced by these viruses differs. We hope that the 
411 data and analysis routines that we make available here will be a valuable resource for such

412 future studies.

\section{METHODS}

414 Reovirus infections of neonatal C57BL/6J mice.

415 Confirmed pregnant female C57BL/6J mice were ordered from Jackson Laboratories to be 416 delivered at embryonic stage E14.5. Litters weighing $3 \mathrm{gram} / \mathrm{pup}$ were gavaged using 417 intramedic tubing (Becton Dickinson 427401) per os with $50 \mu$ with $10^{7}$ PFU reovirus type 1 418 lang (T1L): wildtype or K287T mutant in 1x phosphate buffered saline (PBS) containing green 419 food color (McCormick) via a 1ml tuberculin slip tip syringe (BD 309659) and 30G x 1/2 needle 420 (BD 305106). Litters treated with 1x PBS containing green food color alone on the same day 421 were used as mock controls for the respective infection groups. The mock-infected and 422 reovirus-infected mice pups were weighed daily until the time points used in the study (days 1 , 423 4, 7, and 10). All animal work was conducted ethically, conforming to the U.S. Public Health 424 Service policy, and was approved by the Institutional Animal Care and Use Committee at 425 Cornell University (IACUC Number 2019-0129).

\section{Sample preparation for single-cell transcriptomics of cardiac tissue.}

427 We sacrificed mock-infected and reovirus-infected C57BL/6J mice on day 4, day 7, and day 10 428 post-infection and collected cardiac tissues for single cell transcriptomics. Hearts from 429 respective stages were isolated aseptically, washed with ice-cold Hank's Balanced Salt 430 Solution, HBSS (with calcium and magnesium chloride; Gibco 14025-134), and minced into 1$4312 \mathrm{~mm}$ pieces. Cardiac tissue pieces were then digested in tissue dissociation media with 432 200U/mL collagenase type II (Gibco 17100-015), $1 \mathrm{mg} / \mathrm{ml}$ dispase (Sigma D4693), and 3mM 433 calcium chloride in HBSS for four cycles of 10 minutes under mild agitation at $37^{\circ} \mathrm{C}$ in $1.5 \mathrm{ml}$ eppendorf tubes. After every 10-minute cycle, cell suspension was collected, added to ice-cold 1x PBS with $0.04 \%$ bovine serum albumin (BSA; Sigma A3803) and new dissociation media was added to the tubes. At the end of the digestion, the cells were passed through a $70 \mu \mathrm{m}$ filter and centrifuged into a pellet. To remove most blood contaminants, samples were resuspended in an ammonium-chloride-potassium (ACK) lysis buffer (Lonza \#10-548E) for 3-5 minutes and centrifuged. Samples were then washed again in PBS with $0.04 \%$ BSA and then resuspended at $1 \square \times \square 10^{6}$ cells per ml. Cells from each sample were stained with Trypan Blue and cell viability was calculated on an automated cell counter (Countess II) before loading the cells on 10x Chromium. We used these cell viabilities to adjust the number of cells loaded on 10x Chromium to get the desired number of transcriptomes from viable cells for each sample 444 (5000 cells per sample).

445 Sample preparation for single-cell transcriptomics of intestinal tissue.

We sacrificed mock-infected and reovirus-infected C57BL/6J mice on days 1 and 4 post- 
448 from respective stages were isolated aseptically, washed with ice-cold Hank's Balanced Salt 449 Solution, HBSS (without calcium and magnesium chloride; Gibco 14175-095) to remove 450 contamination. The ileum tissue was then opened longitudinally, washed again with HBSS, 451 and minced into 1-2mm pieces. To isolate the epithelial layer of cells, ileum tissue pieces were 452 incubated in HBSS with 10mM Ethylenediaminetetraacetic acid (EDTA, Invitrogen 15575-038) 453 and $1 \mathrm{mM}$ Dithiothreitol, (DTT, Sigma 43816-10ML) for two cycles of 10 minutes under mild 454 agitation at $37^{\circ} \mathrm{C}$. After every 10 -minute cycle, cell suspension containing the intestinal 455 epithelial cells was collected, added to ice-cold 1x PBS with $0.04 \%$ bovine serum albumin 456 (BSA; Sigma A3803). The undigested pieces of lamina propria were then washed thoroughly 457 with PBS (with calcium and magnesium chloride; Gibco 14080-055) to get rid of all EDTA. 458 These pieces were then transferred to fresh tubes and incubated in $200 \mathrm{U} / \mathrm{ml}$ Collagenase type 459 I (Gibco 17100-017) and 3mM calcium chloride in PBS for three cycles of 10 minutes under 460 mild agitation at $37^{\circ} \mathrm{C}$ in $1.5 \mathrm{ml}$ eppendorf tubes. After every 10-minute cycle, cell suspension 461 containing the lamina propria cells was collected, added to ice-cold phosphate buffered saline, 462 PBS with $0.04 \%$ BSA in separate tubes. At the end of the digestion, the cells were passed 463 through a $40 \mu \mathrm{m}$ filter and washed twice in PBS with $0.04 \%$ BSA and then resuspended at $4641 \square \times \square 10^{6}$ cells per ml. Cells from intestinal epithelium and lamina propria for each sample were 465 stained with Trypan Blue and cell viability was calculated on automated cell counters 466 (Countess II). Cell counts adjusted with viability were then pooled as $40 \%$ epithelial cells and $46760 \%$ lamina propria to adjust the number of cells loaded on 10x Chromium and to get the 468 desired number of transcriptomes from viable cells for each sample (5000 cells per sample).

\section{Single-cell RNA sequencing library preparation.}

470 5000-6000 viable cells per sample (for heart and ileum tissues) were targeted on the 471 Chromium platform (10x Genomics) using one lane per sample per time point. Single-cell 472 libraries were built using the Chromium Next GEM Single Cell 3' Library Construction V3 Kit 473 (10x Genomics) and were then sequenced on an Illumina NextSeq 500 using 75 cycle high 474 output kits (Index $1=8$, Read $1=28$, and Read $2 \square=\square 55$ ) for all samples. Sequencing data 475 were aligned to a combined mouse and reovirus reference genome (described below) using 476 the Cell Ranger 6.0.0 pipeline (10x Genomics).

\section{Sample preparation for Visium spatial transcriptomics.}

478 Whole hearts and intestinal ileum were isolated using aseptic techniques and placed in ice 479 cold sterile Hank's Balanced Salt Solution, HBSS (without calcium and magnesium chloride; 480 Gibco 14175-095). Blood and other contamination were carefully removed by perfusing the 481 tissues with fresh HBSS. Fresh tissues were immediately embedded in Optimal Cutting 482 Compound (OCT) media (SAKURA 25608-930) and frozen in a liquid-nitrogen-cooled 483 isopentane (EMD Millipore, MX0760) bath for spatial transcriptomics experiments. The tissue 484 blocks were cut into $10 \mu \mathrm{m}$ sections using Thermo Scientific CryoStar NX50 cryostat and 485 mounted on Visium Gene Expression slides (10x Genomics), which were pre-cooled to $-20^{\circ} \mathrm{C}$ 486 and used for the Visium Spatial Gene Expression experiment. 
Visium spatial transcriptomics library preparation.

488

489

490

491

492

493

494

495

496

497

498

499

500

501

502

503

504

505

506

507

508

509

510

511

512

513

514

515

516

517

518

519

520

521

522

523

524

525

526

We used the Visium Spatial Gene Expression (10x Genomics) platform for the spatial transcriptomics experiments. Tissue sections from fresh-frozen hearts (mock-infected and reovirus-infected at day 4 and day 7 post infection) and ileum (mock infected and reovirus infected at day 1 and day 4 post infection) were mounted with one section per capture area on individual Visium Gene Expression slides. These sections are then fixed in pre-chilled methanol for 30 minutes and then hematoxylin and eosin (H\&E) stained and imaged, which is later used by the 10x Genomics Space Ranger (version 1.0.0) software to detect the spots which are covered by the tissue. The optimal permeabilization time for $10 \mu \mathrm{m}$ thick sections was found to be 18 minutes for the heart and 12 minutes for the ileum using the 10x Genomics Visium Tissue Optimization kit. Spatially tagged cDNA libraries were built using the 10x Genomics Visium Spatial Gene Expression 3' Library Construction V1 Kit. H\&E-stained heart tissue sections were imaged using Zeiss PALM MicroBeam laser capture microdissection system at 20x objective and the images were stitched and processed using Fiji ImageJ software. cDNA libraries were sequenced on an Illumina NextSeq 500/550 using 150 cycle high output kits (Read $1 \square=28$, Read $2 \square=\square 120$, Index $1 \square=\square 10$, and Index $2 \square=\square 10$ ) for ileum and on an Illumina NextSeq 2K (P2 flow cell) using the 100-cycle kit (Read $1=28$, Read $2=$ 96, Index $1 \square=\square 10$, and Index $2 \square=\square 10$ ). Fiducial frames around the capture area on the Visium slide were aligned manually and spots covering the tissue were selected using Loop Browser 4.0.0 software (10x Genomics). Sequencing data was then aligned to a combined mouse and reovirus reference genome (described below) using the Space Ranger 1.0.0 (10x Genomics) pipeline to derive a feature spot-barcode expression matrix. Visium slide number V19B23-046 was used for spatial transcriptomics experiment on mice hearts (mock-infected 4 dpi: capture area D1, reovirus-infected 4 dpi: capture area B1, mock-infected 7 dpi: capture area $\mathrm{C} 1$, and reovirus-infected 7 dpi: capture area A1). Visium slide number V19B23-045 was used for spatial transcriptomics experiment on mice ileum tissue (mock-infected $1 \mathrm{dpi}$ : capture area D1, reovirus-infected $1 \mathrm{dpi}$ : capture area B1, mock-infected $4 \mathrm{dpi}$ : capture area $\mathrm{C} 1$, and reovirus-infected 4 dpi: capture area $A 1$ ).

\section{Hybridization-based enrichment of viral fragments}

We performed a hybridization-based enrichment of viral fragments on a part of scRNA-seq libraries using XGen NGS target enrichment kit (IDT; 1080577). In this approach, a panel of 5'biotinylated oligonucleotides is used for capture and pulldown of target molecules of interest, which are then PCR amplified and sequenced. We designed a panel of 202 biotinylated probes tiled across the entire reovirus $T 1 \mathrm{~L}$ genome to selectively sequence viral molecules from the scRNA-seq libraries (Supp Table 1). 300ng of fragmented and indexed scRNA-seq libraries from reovirus-WT infected hearts, reovirus-mutant infected hearts, and reovirus-infected ileum were pooled in three separate reactions for xGen hybridization capture. Two rounds of hybridization capture using the $x G e n$ enrichment protocol were performed for every reaction to enrich viral transcripts. Amplification was performed for a total of 18 PCR cycles after the first round of capture. $50 \%$ of the amplified product was used for the second round of hybridization 
527 capture and amplification was performed for a total of 5 PCR cycles after the second round of 528 enrichment. Post-enrichment products were pooled and sequenced on Illumina Mini-seq for 529 ileum libraries and NextSeq 500 for heart libraries.

\section{Reference genome and annotation}

531 Mus musculus genome and gene annotations (assembly: GRCm38) were downloaded from 532 the Ensembl genome browser, and reovirus strain type-1 Lang genome and gene annotations 533 were downloaded and compiled from the NCBI browser. We have shared reovirus genome 534 sequence and annotation files on figshare with the identifier 535 https://doi.org/10.6084/m9.figshare.c.5726372. Genomes were processed using the Cell 536 Ranger v-3.0.0 (10x Genomics) pipeline's mkref command.

537 Single-cell RNAseq data processing and visualization

538 Cells with fewer than 200 unique genes or more than 20 percent of transcripts aligning to mitochondrial genes were removed. The single-cell transcriptomes were log-transformed and normalized using the Scanpy package verison-1.8.1 ${ }^{38}$. We used Scanpy to choose the highly variable genes with min_disp $=0.5$ and max_mean $=3$ thresholds. We then performed mean centering and scaling while regressing out total UMI counts, percent mitochondrial transcripts, $S$ score, and G2M score, followed by principal component analysis (PCA) to reduce the dimensions of the data to the top 20 principal components (PCs). Uniform Manifold Approximation and Projection (UMAP) and the Nearest Neighbor (NN) graph were initialized in this PCA space using the first 20 PCs. The cells were then clustered using the Leiden method with multiple values of clustering resolution to get fine (resolution=0.5) and broad (resolution=0.3) celltype clusters. Cell-type-specific canonical gene markers along with differentially expressed genes (wilcoxon method) for each cluster were used to assign cell type labels. Normalized gene expression was visualized on DotPlots, UMAP plots, and Violin plots across cell type groups. A few cell type clusters representing cell states of the same cell type were grouped into broad cell type groups using cell type marker genes and then used for downstream analysis. Differential gene expression analysis (DGEA) was performed using the rank_gene_groups function in Scanpy with the Wilcoxon statistical method. All gene module scores were calculated using the score_genes function in scanpy.

\section{Reclustering and analysis of endothelial cells, T cells, fibroblasts, and cardiomyocytes}

Normalized gene expression for a specific cell type group was extracted from the combined scRNA-seq dataset. We used Scanpy to reselect the highly variable genes within that cell type group with min_disp=0.5 and max_mean $=3$ thresholds. We then performed mean centering and scaling while regressing out total UMI counts, percent mitochondrial transcripts, S score, and G2M score, followed by principal component analysis (PCA) to reduce the dimensions of the data to the top 20 principal components (PCs). Uniform Manifold Approximation and Projection (UMAP) and the Nearest Neighbor (NN) graph were initialized in this PCA space 
564 using the first 20 PCs. The cells were then reclustered using the Leiden method 565 (resolution=0.5 for endothelial cells, resolution=0.3 for $T$ cells, resolution $=0.2$ for fibroblasts, 566 and resolution=0.3 for cardiomyocytes) to get cell type subclusters. Differentially expressed 567 genes (wilcoxon method) for each subcluster were then used to assign cell subtype labels. 568 Subclusters representing doublets and expressing markers of multiple cell types were then 569 removed from the analysis. Normalized gene expression for differentially expressed genes and 570 genes of interest was visualized on DotPlots and UMAP plots across celltype subgroups. 571 Differential gene expression analysis (DGEA) was performed using the rank_gene_groups 572 function in Scanpy with the Wilcoxon statistical method. All gene module scores were 573 calculated using the score_genes function in Scanpy.

\section{Spatial transcriptomics data processing, integration, analysis, and visualization}

575 Spatial transcriptomics data from barcoded spatial spots from four heart sections were log576 normalized using the Scanpy package (1.8.1). Scanpy package was then used to select highly 577 variable genes for spatial transcriptomics data with min_disp=0.5 and max_mean=3 578 thresholds. We then performed mean centering and scaling while regressing out total UMI 579 counts, percent mitochondrial UMIs, S score, and G2M score, followed by PCA on the spot 580 gene expression matrix, and reduced the dimensions of the data to the top 20 principal 581 components. UMAP and the NN graph were initialized in this PCA space. The spots were then 582 clustered using the Leiden method with multiple values of clustering resolution. The method 583 returned spot clusters representing different tissue regions, which were then visualized on $\mathrm{H} \& \mathrm{E}$ 584 images as spatial transcriptomics maps for individual samples to assign anatomical regions. 585 Normalized gene expression was visualized on spatial transcriptomics maps for all tissue 586 sections. Spot clusters representing the same tissue regions were grouped into broad 587 anatomical region groups using marker genes and then used for downstream analysis. 588 Stereoscope deconvolution method ${ }^{39}$ implemented in the scvi-tools $^{40}$ package was used for 589 integration of spatial transcriptomics data with time-matched scRNA-seq data and cell type 590 prediction values for spatial transcriptomics spots were estimated for the infected heart at 7 591 dpi. DGEA for anatomical regions was performed using the rank_gene_groups function in 592 Scanpy with the Wilcoxon statistical method.

\section{Viral transcript sequencing data processing, filtering, and visualization}

594 Enriched viral transcript data were aligned to a combined mouse and reovirus type-1 Lang 595 genome for all infected samples. Viral unique molecule (UMI) counts were taken from the 596 combined expression matrices and added as metadata in the host gene expression data. Viral 597 UMl counts in empty droplets, droplets with low-quality cells (<200 host UMI counts), droplets 598 with viable cells (>=200 host UMI counts) were sorted by viral UMI and visualized on a 599 histogram to filter out the cell-free ambient viral RNA enriched in the hybridization protocol. 600 Using the distribution of viral UMI counts in empty droplets, thresholds of two viral UMIs and 601 ten viral UMls were used to identify infected cells in the heart and ileum respectively (Supp Fig 
602 2D). Viral transcripts in the infected cells were then visualized on a DotPlot to determine viral 603 tropism in tissues.

604 Gene Ontology term enrichment analysis for scRNA-seq and spatial transcriptomics

605 Gene Ontology (GO) term enrichment analysis was performed on differentially expressed 606 genes using gseapy wrapper package ${ }^{41}$. Differentially expressed genes (two-sided Wilcoxon 607 test, log fold-change threshold $=2.0$, p-value $<10^{-4}$ for scRNA-seq cells, and log fold-change 608 threshold $=0.5$, $\mathrm{p}$-value $<10^{-2}$ for spatial transcriptomics spots) were selected and used for 609 GO term enrichment analysis using GO_Biological_Processes_2021 gene sets in enrichr 610 command $^{42}$. The enriched GO terms of interest were selected and visualized on a BarPlot. The 611 genes associated with GO terms of interested were used to calculate module scores using 612 score_genes command in Scanpy.

\section{ACKNOWLEDGEMENTS}

614 We would like to thank Peter Schweitzer and the Cornell Genomics Center for help with single615 cell and spatial sequencing assays, Cornell Bioinformatics facility for assistance with 616 bioinformatics, and Dr. Danica M. Sutherland from the lab of Dr. Terence S. Dermody at 617 University of Pittsburgh for assistance with animal experiments. We also thank the members of 618 the Parker and De Vlaminck labs for many valuable discussions. This work was supported by 619 R21Al144557 (to J.S.P. and I.D.V.), and DP2AI138242 (to I.D.V.). S.T.C. was supported by 620 the National Institutes of Health and National Institute of Allergy and Infectious Diseases 621 Award T32Al145821.

\section{2}

623

624

625

626

627

628

629

630

631

632

633

634

635

\section{AUTHOR CONTRIBUTIONS}

M.M., J.S.P., and I.D.V. designed the study. M.M., M.M.H., and S.T.C. performed the animal experiments. M.M. and M.M.H. performed the scRNA-seq and spatial transcriptomics experiments. M.M., D.W.M., and M.F.Z.W. analyzed the data. M.M., J.S.P., and I.D.V. wrote the manuscript. All authors provided feedback and comments.

\section{DATA AVAILABILITY}

The authors declare that all sequencing data supporting the findings of this study have been deposited in NCBI's Gene Expression Omnibus (GEO) ${ }^{43}$ with GEO series accession number GSE189636. Raw and processed H\&E-stained tissue images and tissue-spot alignment files matched to spatial transcriptomics datasets have been made publicly available on figshare with identifier https://doi.org/10.6084/m9.figshare.c.5726372 44 . Scripts to reproduce the analysis presented in this study have been deposited on GitHub (https://github.com/madhavmantri/reovirus induced myocarditis).

\section{CONFLICTS}


The authors declare no conflicts.

\section{REFERENCES}

639 1. Pollack, A., Kontorovich, A. R., Fuster, V. \& Dec, G. W. Viral myocarditis-diagnosis, treatment options, and current controversies. Nat. Rev. Cardiol. $2015121112,670-680$ (2015).

$6412 . \quad$ Rose, N. R. Viral Myocarditis. Curr. Opin. Rheumatol. 28, 383 (2016).

642

643

644

645

646

647

648

649

650

651

652

653

654

655

656

657

658

659

660

661

662

663

664

665

666

667

668

669

3. Yajima, T. \& Knowlton, K. U. Viral myocarditis from the perspective of the virus. Circulation 119, 2615-2624 (2009).

4. Tschöpe, C. et al. Myocarditis and inflammatory cardiomyopathy: current evidence and future directions. Nat. Rev. Cardiol. 18, 169-193 (2021).

5. Woodruff, J. F. Viral myocarditis. A review. Am. J. Pathol. 101, 425 (1980).

6. Lasrado, N. \& Reddy, J. An overview of the immune mechanisms of viral myocarditis. Rev. Med. Virol. 30, 1-14 (2020).

7. Sherry, B., Schoen, F. J., Wenske, E. \& Fields, B. N. Derivation and characterization of an efficiently myocarditic reovirus variant. J. Virol. 63, 4840-4849 (1989).

8. Boehme, K. W., Lai, C. M. \& Dermody, T. S. Mechanisms of reovirus bloodstream dissemination. Adv. Virus Res. 87, 1-35 (2013).

9. Guo, Y. et al. The multi-functional reovirus $\sigma 3$ protein is a virulence factor that suppresses stress granule formation and is associated with myocardial injury. PLOS Pathog. 17, e1009494 (2021).

10. Swirski, F. K. \& Nahrendorf, M. Cardioimmunology: the immune system in cardiac homeostasis and disease. Nat. Rev. Immunol. 20181812 18, 733-744 (2018).

11. Sherry, B., Li, X. Y., Tyler, K. L., Cullen, J. M. \& Virgin, H. W. Lymphocytes protect against and are not required for reovirus-induced myocarditis. J. Virol. 67, 6119-6124 (1993).

12. Phillips, M. B., Dina Zita, M., Howells, M. A., Weinkopff, T. \& Boehme, K. W. Lymphatic Type 1 Interferon Responses Are Critical for Control of Systemic Reovirus Dissemination. J. Virol. 95, (2021).

13. Holm, G. H. et al. Interferon Regulatory Factor 3 Attenuates Reovirus Myocarditis and Contributes to Viral Clearance. J. Virol. (2010). doi:10.1128/jvi.01742-09

14. Schaum, N. et al. Single-cell transcriptomics of 20 mouse organs creates a Tabula Muris. Nat. 20185627727 562, 367-372 (2018).

15. Woudstra, L., Juffermans, L. J. M., van Rossum, A. C., Niessen, H. W. M. \& Krijnen, P. A. J. Infectious myocarditis: the role of the cardiac vasculature. Heart Fail. Rev. 23, 583-595 (2018).

16. Chávez-Galán, L., Arenas-Del Angel, M. C., Zenteno, E., Chávez, R. \& Lascurain, R. Cell death mechanisms induced by cytotoxic lymphocytes. Cell. Mol. Immunol. 6, 15-25 (2009). 
670 17. Leuschner, F. et al. Silencing of CCR2 in myocarditis. Eur. Heart J. 36, 1478-1488 (2015).

671 18. Miteva, K. et al. Mesenchymal Stromal Cells Modulate Monocytes Trafficking in Coxsackievirus B3-Induced Myocarditis. Stem Cells Transl. Med. 6, 1249-1261 (2017).

19. Barton, P. J. R. et al. Increased expression of extracellular matrix regulators TIMP1 and MMP1 in deteriorating heart failure. J. Heart Lung Transplant. 22, 738-744 (2003).

675

676

677

678

679

680

681

682

683

684

685

686

687

688

689

690

691

692

693

694

695

696

697

698

699

700

701

702

703

704

705

706

20. Jackson, R. et al. The translation of non-canonical open reading frames controls mucosal immunity. Nature 564, 434-438 (2018).

21. Swertfeger, D. K., Witte, D. P., Stuart, W. D., Rockman, H. A. \& Harmony, J. A. K. Apolipoprotein $\mathrm{J} /$ clusterin induction in myocarditis: A localized response gene to myocardial injury. Am. J. Pathol. 148, 1971-1983 (1996).

22. Szalay, G. et al. Ongoing Coxsackievirus Myocarditis Is Associated with Increased Formation and Activity of Myocardial Immunoproteasomes. Am. J. Pathol. 168, 1542-1552 (2006).

23. Van Der Borght, K. et al. Myocarditis elicits dendritic cell and monocyte infiltration in the heart and self-antigen presentation by conventional type 2 dendritic cells. Front. Immunol. 9, 2714 (2018).

24. Duerr, G. D. et al. Metallothioneins 1 and 2 Modulate Inflammation and Support Remodeling in Ischemic Cardiomyopathy in Mice. Mediators Inflamm. 2016, (2016).

25. Bogomolovas, J. et al. Induction of Ankrd1 in dilated cardiomyopathy correlates with the heart failure progression. Biomed Res. Int. 2015, (2015).

26. Yamada, S. et al. Spatiotemporal single-cell analysis reveals critical roles of mechano-sensing genes at the border zone in remodeling after myocardial infarction. doi:10.21203/rs.3.rs$620498 / v 1$

27. Gupta, S., Markham, D. W., Drazner, M. H. \& Mammen, P. P. A. Fulminant myocarditis. Nat. Clin. Pract. Cardiovasc. Med. 2008 511 5, 693-706 (2008).

28. Mantri, M. \& Scuderi, G. J. Spatiotemporal single-cell RNA sequencing of developing chicken hearts identifies interplay between cellular differentiation and morphogenesis. Github:chicken_heart (2020). doi:https://doi.org/10.5281/zenodo.4517120

29. Asp, M. et al. A Spatiotemporal Organ-Wide Gene Expression and Cell Atlas of the Developing Human Heart. Cell 179, 1647-1660.e19 (2019).

30. Kuppe, C. et al. Spatial multi-omic map of human myocardial infarction. bioRxiv 2020.12.08.411686 (2020). doi:10.1101/2020.12.08.411686

31. Lindner, D. et al. Association of Cardiac Infection With SARS-CoV-2 in Confirmed COVID-19 Autopsy Cases. JAMA Cardiol. 5, 1281-1285 (2020).

32. Bräuninger, H. et al. Cardiac SARS-CoV-2 infection is associated with pro-inflammatory transcriptomic alterations within the heart. Cardiovasc. Res. (2021). doi:10.1093/CVR/CVAB322

33. Lasrado, N., Borcherding, N., Arumugam, R., Starr, T. K. \& Reddy, J. Dissecting the Cellular Landscape and Transcriptome Network in Viral Myocarditis by Single-Cell RNA Sequencing. 
34. Baty, C. J. \& Sherry, B. Cytopathogenic effect in cardiac myocytes but not in cardiac fibroblasts is correlated with reovirus-induced acute myocarditis. J. Virol. 67, 6295-6298 (1993).

35. Stewart, M. J., Blum, M. A. \& Sherry, B. PKR's protective role in viral myocarditis. Virology 314, 92-100 (2003).

36. Miyamoto, S. D. et al. Cardiac Cell-specific Apoptotic and Cytokine Responses to Reovirus Infection: Determinants of Myocarditic Phenotype. J. Card. Fail. 15, 529-539 (2009).

37. Stewart, M. J., Smoak, K., Blum, M. A. \& Sherry, B. Basal and Reovirus-Induced Beta Interferon (IFN- $\beta$ ) and IFN- $\beta$-Stimulated Gene Expression Are Cell Type Specific in the Cardiac Protective Response. J. Virol. 79, 2979-2987 (2005).

38. Wolf, F. A., Angerer, P. \& Theis, F. J. SCANPY: Large-scale single-cell gene expression data analysis. Genome Biol. 19, 1-5 (2018).

39. Andersson, A. et al. Single-cell and spatial transcriptomics enables probabilistic inference of cell type topography. Commun. Biol. 202031 3, 1-8 (2020).

40. Gayoso, A. et al. scvi-tools: a library for deep probabilistic analysis of single-cell omics data. bioRxiv 2021.04.28.441833 (2021). doi:10.1101/2021.04.28.441833

41. Subramanian, A. et al. Gene set enrichment analysis: A knowledge-based approach for interpreting genome-wide expression profiles. Proc. Natl. Acad. Sci. 102, 15545-15550 (2005).

42. Xie, Z. et al. Gene Set Knowledge Discovery with Enrichr. Curr. Protoc. 1, e90 (2021).

43. Edgar, R. Gene Expression Omnibus: NCBI gene expression and hybridization array data repository. Nucleic Acids Res. (2002). doi:10.1093/nar/30.1.207

44. Mantri, M. Reovirus-induced-myocarditis. (2021).

doi:https://doi.org/10.6084/m9.figshare.c.5726372 